\title{
An automatic stopping criterion for nonlinear anisotropic diffusion
}

\author{
César Bustacara-Medina \\ cbustaca@javeriana.edu.co \\ Pontificia Universidad Javeriana \\ Bogotá D.C., Colombia
}

\author{
Leonardo Flórez-Valencia \\ florez-1@javeriana.edu.co \\ Pontificia Universidad Javeriana \\ Bogotá D.C., Colombia
}

\begin{abstract}
Nonlinear anisotropic diffusion (NAD) filtering is a procedure based on nonlinear evolution PDEs which seeks to improve images qualitatively by removing noise while preserving details and even enhancing edges. However, well-known implementations are sensitive to parameters which are necessarily tuned to sharpen a narrow range of edge slopes; otherwise, edges are either blurred or staircased [Kee2002]. One important parameter is the iterations number, for that reason, in this paper a stopping criterion to halt the diffusion process is proposed. To meet this goal, two stopping criteria were compared. The first is the stopping criterion proposed by Joao et. al. [Joã2016], which is based on the Mean Squared Error (MSE). The second is our proposed method based on the CIRR contrast measure. To this end, a comparative analysis of five diffusion methods is performed. Four of them are nonlinear anisotropic diffusion methods and the fifth is the Perona-Malik method. According to the tests performed, the number of iterations required by the smoothing algorithms using the proposed stopping criterion is lower.
\end{abstract}

Keyword: Smoothing, Stopping criteria, Nonlinear anisotropic diffusion, Edge detection

\section{INTRODUCTION}

Medical images typically suffer from one or more of the following imperfections, low resolution (in the spatial and spectral domains), high level of noise, low contrast, geometric deformations and/or presence of imaging artifacts. These imperfections can be inherent to the imaging modality (e.g., X-rays offer low contrast for soft tissues, ultrasound produces very noisy images, and metallic implants will cause imaging artifacts in $\mathrm{CT}$ ) or the result of a deliberate trade-off during acquisition. For example, finer spatial sampling may be obtained through a longer acquisition time. However that would also increase the probability of patient movement and thus blurring. To remove noise while preserving details and even enhancing edges techniques based on Partial Differential Equations (PDEs) have been used. The idea of using the PDE diffusion equations in image denoising and restoration arose from the use of the Gaussian filter in multiscale image analysis.

Permission to make digital or hard copies of all or part of this work for personal or classroom use is granted without fee provided that copies are not made or distributed for profit or commercial advantage and that copies bear this notice and the full citation on the first page. To copy otherwise, or re- publish, to post on servers or to redistribute to lists, requires prior specific permission and/or a fee.
Convolving an image with a two or three dimensional Gaussian filter is equivalent to the solution of the diffusion equation in two or three dimensions [Bar2014]. Nowadays, PDEs have been successfully applied to many problems in image processing and computer vision [Ter1994, Cas1998, Sap2006, Aub2006, Cao2003], e.g., denoising [Per1990a], enhancement [Rud1989], inpainting [Ber2000], segmentation [Li2005], stereo and optical flow computation [Sap2006].

Nonlinear anisotropic diffusion is a variant of the heat equation, generalized in two regards: nonlinearity and anisotropy. Nonlinearity in diffusion means that diffusion tensors are automatically generated from the processed image. Anisotropy means that the smoothing induced by the PDE can be favored in some directions and prevented in others. This is specified by local eigenvectors and eigenvalues of the diffusion tensor field [Wei1996]. Diffusion coefficients are thus location and direction dependent, generalizing the approach of Perona and Malik [Per1990, Per1990a] which is only location dependent.

NAD is a powerful image processing technique, which allows to simultaneously remove the noise and enhance sharp features in two or three dimensional images. Anisotropic diffusion is understood here in the sense of Weickert [Wei1998], meaning that diffusion tensors are anisotropic and reflect the local orientation of image features. Weickert [Wei1999] proposed two nonlinear anisotropic diffusion algorithms. The first one is called Edge Enhancing Diffusion (EED), which al- 
lows smoothing while preserving the edges. The second one is called Coherence Enhancing Diffusion (CED), which allows smoothing based on the structures (flowlike) present in the images. Based on the filters defined by Weicket, several methods have been proposed. For example, Bazan et. al. [Baz2007, Baz2009] proposed a new approach based on nonlinear anisotropic diffusion and bilateral filtering for electron tomography of mitochondria. Dong et. al. [Don2015] introduced a source term in the CED filter to restore the initial image and contrast lost by pure diffusion filters. Prasath [Pra2016] proposed an adaptive coherence enhancement diffusion filter (CED) combining anisotropic diffusion and diffusion functions derived from the structural tensor. Mirebeau et. al. [Mir2014] proposed two variants to the Weickert's algorithms. The first is associated with the EED algorithm, which is called Conservative variant of EED (cEED). The second is called Conservative variant of CED (cCED). The main distinction lies in the definition of the diffusion parameters of the diffusion tensor.

This paper is organized as follows: In section 2, nonlinear anisotropic diffusion filters are explained. In section 3, the most relevant methods to stop the diffusion propagation are described. In section 4, the proposed method for stopping the propagation is briefly explained. In section 5 , tests performed in $2 \mathrm{D}$ and $3 \mathrm{D}$ images are presented, and the paper finishes with some conclusions.

\section{NONLINEAR ANISOTROPIC DIFFUSION FILTERS}

The idea of nonlinear anisotropic diffusion was pioneered by Nitzbeg et. al. [Nit1992] and Cottet et al. [Cot1993]. Later on, Weickert [Wei1999] put forward a formal method for enhancing the elongated structure, referred to as coherence-enhanced diffusion (CED).

NAD filtering is a procedure based on nonlinear evolution PDEs which seeks to improve images qualitatively by removing noise while preserving details and even enhancing edges. In the anisotropic case not only the amount of diffusion is adapted locally to the data but also the direction of smoothing. It allows for example to smooth along image edges while inhibiting smoothing across edges. This can be achieved by replacing the scalar-valued diffusivity function by a matrix-valued diffusion tensor [Bro2006].

The eigenvectors of the diffusion tensor define the principal directions of smoothing and the corresponding eigenvalues define the amount of smoothing. Weickert based the diffusion tensor on the structure tensor [Wei1996, Wei1997, Wei1998], which describes struc- tures in the image using first order derivative information [Bus2016].

In general, any nonlinear anisotropic diffusion can be described by the equation

$$
\frac{\partial u}{\partial t}=\operatorname{div}(D(\nabla u) \nabla u)
$$

where $u$ is the initial smoothed image that is initialized with the input image $f$ (that is $u(\mathbf{x}, 0)=f(\mathbf{x})$ ), and $D$ represents a matrix-valued diffusion tensor that describes the smoothing directions and the corresponding diffusivities [Erd2012]. In this case, the diffusion tensor $D$ is a function of $\mathbf{x}$, i.e., depends on the space. Additionally, $D$ is a positive definite symmetric matrix [Wei1998, Wei2002]. The idea is to adaptively choose the diffusion coefficient $D$ such that intra-regions become smooth while edges of inter-regions are preserved [Cha2010]. As $D$ must be a nonnegative function of gradient magnitude so that small variations in intensity such as noise or shading can be well smoothed, and edges with large intensity transition are retained. It is generally given by an exponential function or an inverse quadratic function, and determined by the gradient magnitude with respect to a predetermined edge strength threshold [Cha2010].

Thus the given image $u$ is usually convolved with a Gaussian kernel $G_{\sigma}$ with a relatively small standard deviation $\sigma$ as a presmoothing step. Cottet and Germain [Cot1993] and Weickert [Wei1996] devise a diffusivity matrix of the form:

$$
D_{\sigma}=\left[\begin{array}{lll}
v_{1} & v_{2} & v_{3}
\end{array}\right]\left[\begin{array}{ccc}
\lambda_{1} & 0 & 0 \\
0 & \lambda_{2} & 0 \\
0 & 0 & \lambda_{3}
\end{array}\right]\left[\begin{array}{c}
v_{1}^{T} \\
v_{2}^{T} \\
v_{3}^{T}
\end{array}\right]
$$

where the vectors $v_{i}$ are the eigenvectors of the structure tensor and the parameters $\lambda_{i}$ are functions of the eigenvalues of the structure tensor. The images's structure tensor is defined as [Wei1997]:

$$
J_{\rho}\left(\nabla u_{\sigma}\right)=G_{\rho} *\left(\nabla u_{\sigma} \cdot \nabla u_{\sigma}^{T}\right)
$$

where $G_{\rho}$ is the Gaussian kernel with standard deviation $\rho$ (integration scale), over which the orientation information is averaged, and $\nabla u_{\sigma}$ is the gradient of the image $u$ at scale $\sigma$. Principle axis transformation gives the eigenvectors and eigenvalues of $J_{\rho}\left(\nabla u_{\sigma}\right)$ [Men2009]. 


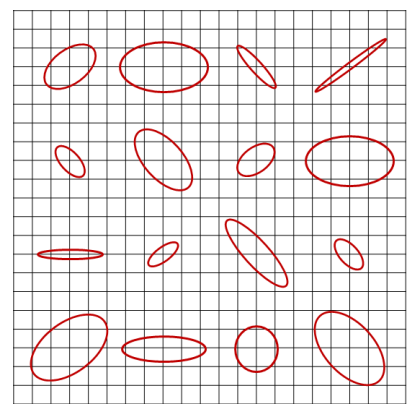

Figure 1: Nonlinear anisotropic diffusion (Adapted from [Li2005a])

Figure 1 shows a nonlinear anisotropic diffusion process illustrated by ovals of different sizes and different orientations. The ratio of two dimensions of the ovals can be arbitrarily different. The orientations of the ovals can also be random. This is the diffusion filter with the ultimate freedom in terms of the changes of filter strength location-wise or direction-wise [Li2005a].

Two specializations of nonlinear anisotropic diffusion were introduced by Weickert, edge-enhancing diffusion (EED) and coherence-enhancing diffusion (CED) [Wei1998]. Both were initially defined in two dimensions. EED was designed to smooth noise while enhancing edges and CED was designed to enhance line-like textures. CED is essentially one dimensional diffusion [Wei1999], since there is either diffusion in one direction or almost not diffusion at all. In addition, Mirebeau et. al. [Mir2014] proposed a conservative variant of both the EED and CED method. These variants are called cEED and cCED respectively.

\section{DIFFUSION STOPPING CRI- TERIA}

Filtering process involves the solution of the anisotropic diffusion equations as a time-marching problem, a possible approach is to halt the filtering when a certain set of metrics falls below a predefined threshold [Joã2016]. In addition, the definition of the number of iterations (diffusion time $t$ ) based on the metrics selected to stop the diffusion process is crucial to obtain a good image reconstruction [Baz2007]. For example, if $t$ is too small, the reconstructed signal is very noisy; if $t$ is too large it is smooth and discontinuities are lost. In conclusion, automatically stopping the diffusion process is a challenging task. Normally, the stopping criterion depends on the image characteristics and on the parameters of the diffusion equation.
Several authors have addressed this issue in the past in an attempt to devise an optimal stopping criterion [Baz2007, Ily2010]. A brief review of previous works on the stopping criteria is presented below.

- Sporring and Weickert [Spo1999]

This is focused on the maximal entropy change by scaling to estimate the size of image structures. They argued that the minimal change by scale indicates especially stable scales with respect to evolution time, and conjectured that these scales could be good candidates for stopping times in nonlinear diffusion processes. In addition, this is based on the signal to noise ratio (SNR) and the relative variance at time $t$ and the initial image [Ily2010]. The authors pointed out that the monotonically decreasing 'relative variance', $0 \leq \operatorname{var}(u) / \operatorname{var}\left(u_{0}\right) \leq$ 1 , could be used to measure the distance of $u$ from the initial state $u_{0}$ and, by prescribing an appropriate value for the relative variance, it can constitute a good criterion for stopping the nonlinear diffusion [Baz2007].

- Capuzzo and Ferretti [Cap2001]

They determine the optimal time by finding the minimum of a performance index which balances the computing and stopping costs. This is then applied to the regularized Perona-Malik equation. Their method requires a constant that is found by experimentation using a typical image with similar details and discontinuities as the image to be processed. This is a rather vague requirement and they demonstrate that one only needs some approximation to the constant [Ily2010].

- Mrázek and Navara [Mra2003]

They choose the stopping criteria so that the correlation of the signal $u(T)$ and noise $u(0)-u(T)$ in the filtered image is minimized. This method is applicable to any images where the noise to be removed is uncorrelated with the signal, under the assumptions that the filter used is suitable for the given type of data, and that neither the additive noise nor the filtering procedure alter the average gray value; no other knowledge (e.g. the noise variance, training data etc.) is needed [Mra2003]. This method is applied to several nonlinear filters both isotropic and anisotropic [Ily2010]. In addition, this requires no prior estimation of the noise statistics [Tsi2013].

Proposed method is called decorrelation criterion. This selects the time $\mathrm{T}$ as the time that minimizes the correlation 


$$
T \equiv \arg \min _{t} \frac{\operatorname{cov}(u(0)-u(t), u(t))}{\sqrt{\operatorname{var}(u(0)-u(t)) \cdot \operatorname{var}(u(t))}}
$$

- Gilboa, Sochen and Zeevi [Gil2004]

Stopping criterion is based on obtaining of minimal SNR, i.e. one stops the process when filtering more signal than noise. This is done by estimating the covariance of the image and the noise. This method requires an estimate of the standard deviation of the noise $\sigma_{n 0}$ of the input noisy image $u(0)$, which is considered to be a priori known [Tsi2013]. They also compare the advantages and disadvantages of the approaches that use the covariance [Ily2010].

The condition for selecting the value of parameter $\mathrm{T}$ is

$$
T=\arg \min _{t} \frac{\partial_{t} \operatorname{cov}(\bar{N}, u(0)-u(t))}{\partial_{t} \operatorname{var}(u(0)-u(t))}
$$

The variance of noise $\bar{N}$ of the original image is considered a priori known.

- Bazán and Blomgren [Baz2007]

This stopping criterion is inspired by observation of the behavior of the correlation between the noisefree image and the filtered image, $\operatorname{corr}(f, u)$, and the correlation between the noisy image and the filtered image, $\operatorname{corr}\left(u_{0}, u\right)$. Although the former measure is only available in experimental settings it helps validate the usefulness of the latter.

The nonlinear diffusion process starts from the observed (noisy) image, $u_{0}(x)$, and creates a set of filtered images, $u(x, t)$, by gradually removing noise and details from scale to scale until, as $t \rightarrow \infty$, the image converges to a constant value. During this process the correlation between the noise-free image and the filtered image increases as the filtered image moves closer to the noise-free image. This behavior continues until it reaches a peak from where the measure decreases as the filtered image moves slowly towards a constant value. During the same process the correlation between the noisy image and the filtered image decreases gradually from a value of 1.0 (perfect correlation), to a constant value, as the filtered image becomes smoother [Baz2009].

By comparing both measures, they observed that as $\operatorname{corr}(f, u)$ reaches its maximum (the best possible reconstructed image), the curvature of $\operatorname{corr}\left(u_{0}, u\right)$ changes sign. They suggested that a good stopping point of the diffusion process is where the second derivative of $\operatorname{corr}\left(u_{0}, u\right)$ reaches a maximum [Baz2009].
- Tsiotsios and Petrou [Tsi2013]

The method examines directly the quality of the edges in every iteration. It evaluates, in every iteration, the quality of a percentage of the true edges of the image, taking into consideration the contrast and the noise brightness fluctuations around them, and leads to a judicious choice of the stopping time $T$ that corresponds to the maximum overall quality of the edges [Tsi2013]. This method requires an estimate of the standard deviation of the noise $\sigma_{n 0}$ of the input noisy image $u(0)$, which is considered to be a priori known [Tsi2013]. The proposed method has five steps that finally compute the stop time $T$ as

$$
T=\arg \max _{t} \frac{1}{N} \sum_{i=1}^{N} Q_{i}(t)
$$

where $\mathrm{N}$ is the number of edges and $Q(t)$ reflects the quality of the edges within the image, in every iteration.

- Joao, Gambaruto, Tiago and Sequeira [Joã2016]

The relative residual error of Mean Square Error (MSE) measure is the metric chosen for this purpose, specifically

$$
\frac{\left|M S E_{t+1}-M S E_{t}\right|}{\left|M S E_{t+1}\right|}<\varepsilon_{1}
$$

where $\varepsilon_{1}=10^{-2}$. The choice of $\varepsilon_{1}$ is influenced by the need for a small value to identify a convergence of solution, and large enough to make the iterative procedure less computationally demanding.

In addition, they propose to use Structural Similarity Index Metric (SSIM) in combination with above criterion, using a threshold value of $S S I M<\varepsilon_{2}$ and $\varepsilon_{2}=0.7$. The choice of $\varepsilon_{2}$ is influenced by the importance of allowing the image to evolve and deviate from the original, and yet not to allow too large a distortion that will make the image unrecognizable compared to the original.

In conclusion, the optimal number of iterations is obtained when $\left|M S E_{t+1}-M S E_{t}\right| /\left|M S E_{t+1}\right|<$ $10^{-2}$ and $\operatorname{SSIM}_{\beta}(t+1)<0.7$.

This, depending on the size of each image and respective data set, can be rather computationally expensive; therefore, a parallel implementation was used, which proved to be effective. 


\section{STOPPING PROPOSED \\ CRITERION}

Sporring and Weickert pointed out that the monotonically decreasing 'relative variance', $0 \leq$ $\operatorname{var}(u) / \operatorname{var}\left(u_{0}\right) \leq 1$, could be used to measure the distance of $u$ from the initial state $u_{0}$ and, by prescribing an appropriate value for the relative variance, it can constitute a good criterion for stopping the nonlinear diffusion.

The Contrast Improvement Ratio Revisited measure (CIRR) [Bus2019] is an increasing function that reaches its steady state when $t \rightarrow \infty$. The residual error of the CIRR measure is a decreasing function of values between one and zero. Then, applying the same idea presented by Sporring and Weickert, it can be indicated that the residual error of the CIRR measure can be used as a stopping criterion to halt diffusion processes.

The residual error of CIRR measure is computed as:

$$
C I R R \text { index }_{t}=\frac{\left|C I R R_{t}-C I R R_{t-1}\right|}{\left|C I R R_{t}\right|}
$$

where $t$ is the diffusion time. Diffusion process is iterated while CIRR index $x_{t}$ is greater that a specific constant $\varepsilon$ defined by the user. In general, the stopping criteria is defined as:

$$
\frac{\left|C I R R_{t}-C I R R_{t-1}\right|}{\left|C I R R_{t}\right|}>\varepsilon
$$

The choice of $\varepsilon$ is influenced by the need for a small value to identify a convergence of solution, and large enough to make the iterative procedure less computationally demanding. According to João et. al. [Joã2016], who defined a stopping criterion for anisotropic diffusion, $\varepsilon$ value can be $10^{-2}$.

\section{EXPERIMENTAL RESULTS}

Nonlinear anisotropic diffusion algorithms require several parameters such as diffusion time, lambda, noise scale and feature scale. Therefore, to select the most appropriate diffusion algorithm to preserve the edge information is a complex task. For this reason, the experiments were divided into two parts. The first part is related to the automatic definition of the diffusion time for both two-dimensional and three-dimensional images using the stopping criterion presented by Joao et. al. [Joã2016] and the proposed criterion. The second part is associated with the selection of the algorithm that generates better results with respect to the image quality measures as Mean Square Error (MSE), Peak Signal-Noise
Ratio (PSNR), and Contrast Improvement Ratio Revisited (CIRR). As a qualitative measure of smoothing, the Canny edge detection filter is used [Can1986, Afr2017]. The filter is applied to the resulting images by using each of the stopping criteria.

\subsection{D Case}

In the first part, the stopping criterion proposed by Joao et. al. [Joã2016] is based on the MSE quality measure, this criterion is called JGTS. The proposed criterion is based on the CIRR measure and it is called BF. These two stopping criteria are evaluated.

\subsubsection{Original Images}

Five images were selected that are used traditionally in image processing. The images are the baboon, barbara, boat, cameraman and lena. Each of them has different characteristics that allow evaluating the quality of the smoothing obtained for each of them according to each stopping criterion.

Initially smoothing is calculated using each of the selected diffusion algorithms (Isotropic, CED, cCED, EED, cEED). The number of iterations (diffusion time) applied is initially set to 10 . In each iteration the MSE, PSNR and CIRR quality measures are calculated. The results for the lena image are presented in Tables 1,2 and 3 respectively.

As you can see in Tables 1, 2, and 3 respectively, the values for the CED, cCED and EED algorithms are quite similar for the MSE measure. In the case of PSNR, the values are very similar in all cases. For the CIRR measure, the values obtained by using CED, cCED and cEED are similar. However, in the latter case, the cEED algorithm is reduced compared to CED and cCED, as the number of iterations increases.

\begin{tabular}{|c|c|c|c|c|c|}
\hline Iter & Isotropic & CED & cCED & EED & cEED \\
\hline 1 & 2,870 & 3,731 & 3,171 & 7,513 & 4,652 \\
\hline 2 & 4,127 & 8,146 & 7,044 & 12,618 & 7,082 \\
\hline 3 & 5,055 & 12,017 & 10,623 & 16,728 & 8,915 \\
\hline 4 & 5,875 & 15,537 & 13,935 & 20,255 & 10,498 \\
\hline 5 & 6,650 & 18,676 & 16,870 & 23,364 & 11,952 \\
\hline 6 & 7,407 & 21,500 & 19,628 & 26,176 & 13,322 \\
\hline 7 & 8,160 & 24,122 & 22,142 & 28,750 & 14,650 \\
\hline 8 & 8,916 & 26,491 & 24,437 & 31,136 & 15,942 \\
\hline 9 & 9,671 & 28,692 & 26,626 & 33,370 & 17,203 \\
\hline 10 & 10,427 & 30,790 & 28,647 & 35,482 & 18,442 \\
\hline
\end{tabular}

Table 1: MSE measure - lena. 


\begin{tabular}{|c|c|c|c|c|c|}
\hline Iter & Isotropic & CED & cCED & EED & cEED \\
\hline \hline 1 & 43,205 & 42,065 & 42,771 & 39,025 & 41,107 \\
\hline 2 & 41,627 & 38,674 & 39,305 & 36,774 & 39,282 \\
\hline 3 & 40,747 & 36,986 & 37,521 & 35,549 & 38,282 \\
\hline 4 & 40,093 & 35,870 & 36,342 & 34,718 & 37,572 \\
\hline 5 & 39,555 & 35,070 & 35,512 & 34,098 & 37,009 \\
\hline 6 & 39,087 & 34,459 & 34,855 & 33,604 & 36,538 \\
\hline 7 & 38,666 & 33,959 & 34,331 & 33,197 & 36,125 \\
\hline 8 & 38,282 & 33,552 & 33,903 & 32,851 & 35,758 \\
\hline 9 & 37,929 & 33,206 & 33,530 & 32,550 & 35,427 \\
\hline 10 & 37,602 & 32,899 & 33,213 & 32,283 & 35,125 \\
\hline
\end{tabular}

Table 2: PSNR measure - lena.

\begin{tabular}{|c|c|c|c|c|c|}
\hline Iter & Isotropic & CED & cCED & EED & cEED \\
\hline \hline 1 & 0,00016 & 0,00031 & 0,00026 & 0,00055 & 0,00030 \\
\hline 2 & 0,00027 & 0,00072 & 0,00061 & 0,00106 & 0,00052 \\
\hline 3 & 0,00036 & 0,00110 & 0,00096 & 0,00149 & 0,00070 \\
\hline 4 & 0,00044 & 0,00145 & 0,00129 & 0,00186 & 0,00086 \\
\hline 5 & 0,00051 & 0,00177 & 0,00158 & 0,00218 & 0,00101 \\
\hline 6 & 0,00058 & 0,00205 & 0,00185 & 0,00246 & 0,00114 \\
\hline 7 & 0,00065 & 0,00230 & 0,00210 & 0,00272 & 0,00127 \\
\hline 8 & 0,00071 & 0,00253 & 0,00232 & 0,00296 & 0,00140 \\
\hline 9 & 0,00078 & 0,00274 & 0,00253 & 0,00317 & 0,00153 \\
\hline 10 & 0,00084 & 0,00294 & 0,00273 & 0,00337 & 0,00165 \\
\hline
\end{tabular}

Table 3: CIRR measure - lena.

Figures 2, 3, and 4 show the behavior of the three measures for each of the smoothing algorithms used. The best results are obtained using the Isotropic and cEED algorithms for all cases.

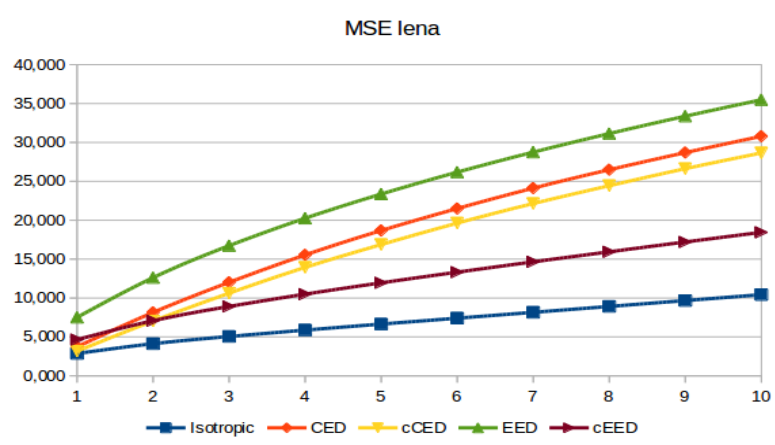

Figure 2: MSE measure - lena.

Table 4 shows the total number of iterations defined by the stopping criteria JGTS and BF for the five reference images. As you can see, the results are quite similar, they differ in one or two iterations. It can also be observed that the isotropic and cEED methods require a greater number of iterations. This is directly related to the MSE, PSNR and CIRR quality measurements obtained.

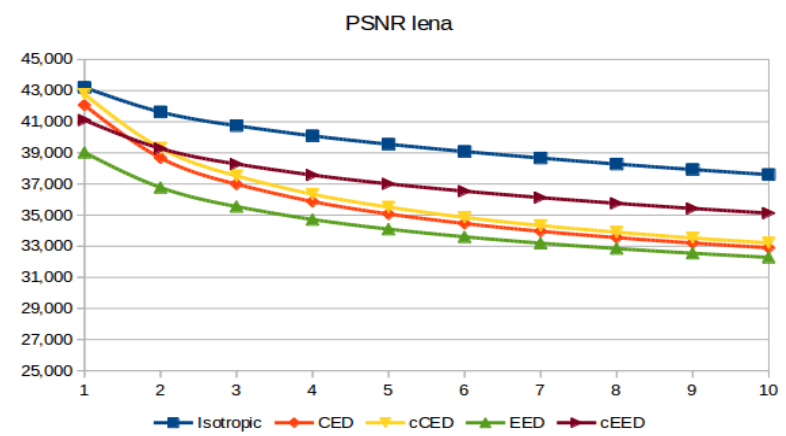

Figure 3: PSNR measure - lena.

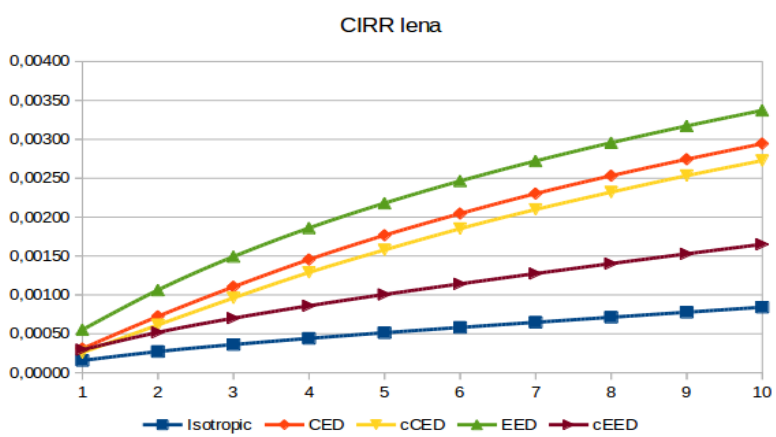

Figure 4: CIRR measure - lena.

\begin{tabular}{|c|c|c|c|c|c|c|}
\hline Image & Index & Iso & CED & cCED & EED & cEED \\
\hline \hline \multirow{3}{*}{ baboon } & $\mathrm{JGTS}$ & 10 & 6 & 6 & 5 & 8 \\
\cline { 2 - 7 } & $\mathrm{BF}$ & 10 & 6 & 7 & 6 & 9 \\
\hline \multirow{3}{*}{ barbara } & $\mathrm{JGTS}$ & 10 & 6 & 7 & 6 & 9 \\
\cline { 2 - 7 } & $\mathrm{BF}$ & 10 & 6 & 6 & 6 & 9 \\
\hline \multirow{3}{*}{ boat } & $\mathrm{JGTS}$ & 8 & 7 & 7 & 7 & 7 \\
\cline { 2 - 7 } & $\mathrm{BF}$ & 10 & 7 & 8 & 7 & 9 \\
\hline \multirow{3}{*}{ cameraman } & $\mathrm{JGTS}$ & 8 & 7 & 7 & 6 & 7 \\
\cline { 2 - 7 } & $\mathrm{BF}$ & 9 & 6 & 7 & 6 & 8 \\
\hline \multirow{2}{*}{ lena } & $\mathrm{JGTS}$ & 6 & 7 & 7 & 6 & 6 \\
\cline { 2 - 7 } & $\mathrm{BF}$ & 7 & 7 & 7 & 6 & 7 \\
\hline
\end{tabular}

Table 4: Number of iterations using JGTS and BF stopping criteria.

In the particular case of lena image, Tables 5 and 6 show the values for the MSE and CIRR measurements respectively. It can be indicated that the isotropic method and cEED are those that present a variation. For these 
two cases, the number of iterations required by the proposed method $(\mathrm{BF})$ is greater than for the JGTS method.

\begin{tabular}{|c|c|c|c|c|c|}
\hline Iter & Isotropic & CED & cCED & EED & cEED \\
\hline \hline 1 & 1,0000 & 1,0000 & 1,0000 & 1,0000 & 1,0000 \\
\hline 2 & 0,3047 & 0,5420 & 0,5498 & 0,4046 & 0,3432 \\
\hline 3 & 0,1835 & 0,3221 & 0,3369 & 0,2457 & 0,2057 \\
\hline 4 & 0,1396 & 0,2266 & 0,2377 & 0,1741 & 0,1507 \\
\hline 5 & 0,1165 & 0,1681 & 0,1740 & 0,1331 & 0,1217 \\
\hline 6 & $\mathbf{0 , 1 0 2 2}$ & 0,1314 & 0,1405 & $\mathbf{0 , 1 0 7 4}$ & $\mathbf{0 , 1 0 2 9}$ \\
\hline 7 & 0,0923 & $\mathbf{0 , 1 0 8 7}$ & $\mathbf{0 , 1 1 3 6}$ & 0,0895 & 0,0907 \\
\hline 8 & 0,0848 & 0,0894 & 0,0939 & 0,0767 & 0,0810 \\
\hline
\end{tabular}

Table 5: MSE index - lena.

\begin{tabular}{|c|c|c|c|c|c|}
\hline Iter & Isotropic & CED & cCED & EED & cEED \\
\hline \hline 1 & 1,0000 & 1,0000 & 1,0000 & 1,0000 & 1,0000 \\
\hline 2 & 0,4206 & 0,5728 & 0,5798 & 0,4801 & 0,4308 \\
\hline 3 & 0,2502 & 0,3440 & 0,3606 & 0,2880 & 0,2599 \\
\hline 4 & 0,1793 & 0,2412 & 0,2541 & 0,1976 & 0,1854 \\
\hline 5 & 0,1411 & 0,1765 & 0,1837 & 0,1465 & 0,1447 \\
\hline 6 & 0,1172 & 0,1363 & 0,1474 & $\mathbf{0 , 1 1 5 6}$ & 0,1194 \\
\hline 7 & $\mathbf{0 , 1 0 1 7}$ & $\mathbf{0 , 1 1 1 5}$ & $\mathbf{0 , 1 1 7 6}$ & 0,0946 & $\mathbf{0 , 1 0 3 7}$ \\
\hline 8 & 0,0913 & 0,0907 & 0,0961 & 0,0793 & 0,0915 \\
\hline
\end{tabular}

Table 6: CIRR index - lena.

Figures 5 and 6 reveal a variation in the behavior of the MSE index between iterations 2 and 3 for the isotropic and cEED methods. This may be the reason why the BF method is more uniform in the number of iterations required to stop the diffusion process.

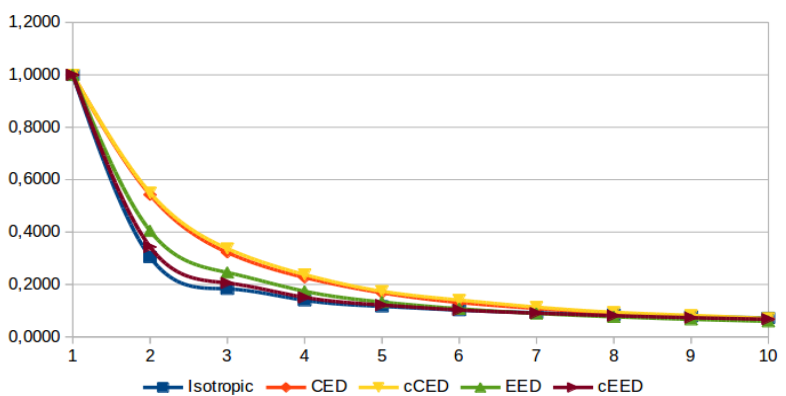

Figure 5: MSE index behaviour for lena image.

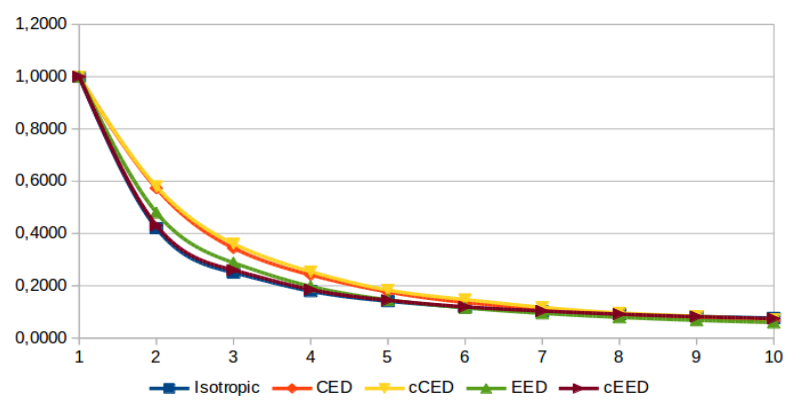

Figure 6: CIRR index behaviour for lena image.

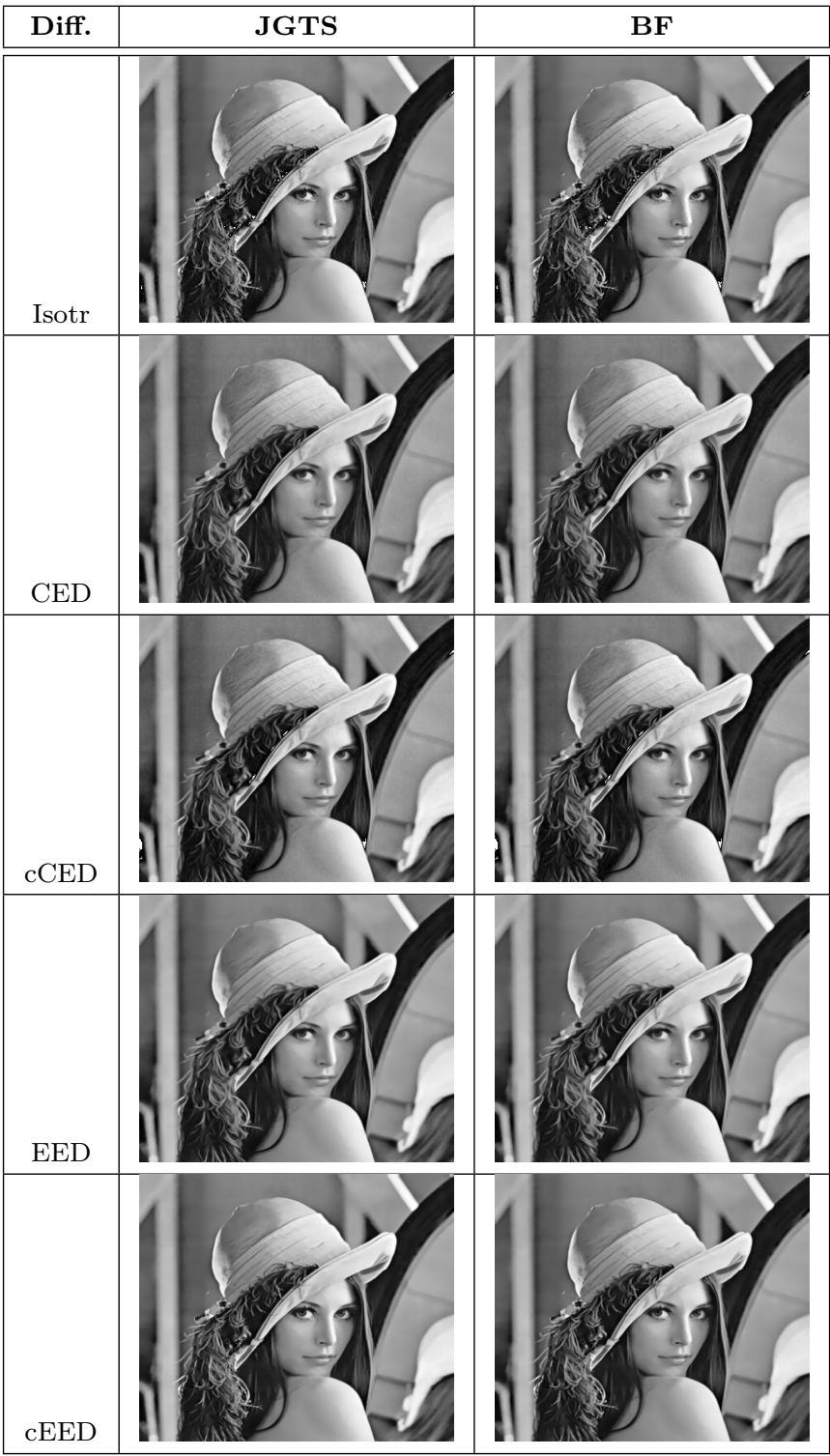

Figure 7: Smoothed image of lena using JGTS and BF stopping criteria. 
Table 7 shows the images obtained by applying the selected smoothing methods (Isotropic, CED, cED, EED and $\mathrm{cED}$ ). Based on the results for the MSE and CIRR measures, the results are the same or similar. Subjectively, it is very difficult to notice the differences.

After exploring the lena image content, the eye region was identified to zoom in and see in a greater level of detail the effect of the smoothing algorithms. Table 8 presents the original image and the images obtained when applying the three algorithms that show a better behaviour with respect to the selected quality measures. As can be seen, the original image differentiates a semi-circular region in the centre of the eye. This region is maintained when applying isotropic smoothing and cEED, however, when cCED smoothing is applied, that region becomes blurred. The latter behaviour is maintained when applying the CED and EED algorithms (see Table 8).

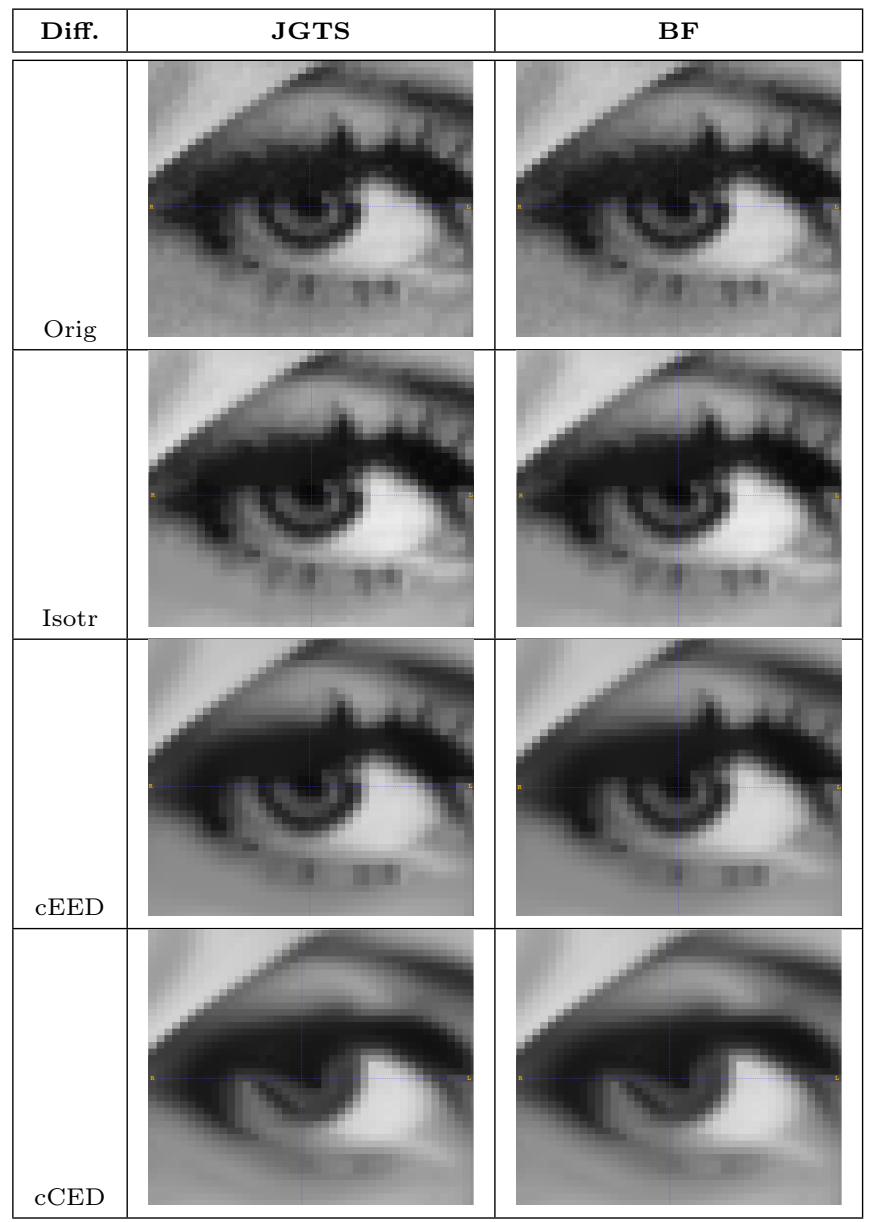

Figure 8: Zoom in of eye region of smoothed image of lena.

In addition, it is observed that the isotropic algorithm presents a lower smoothing in some regions compared to the cEED and cCED algorithms, for example, in the upper left region of the images, it is seen that the isotropic algorithm presents a more stepped variation than the results of the cEED and cCED algorithms.

To visually identify the impact of the smoothing algorithms, row 266 of the lena image was selected. Figure 9 shows the behaviour of the original image and the images obtained from applying each smoothing algorithms. As can be seen, the isotropic diffusion algorithm generates a profile very close to the original image and therefore the image quality measures are better. The cEED algorithm maintains the intensity in the areas where edges are present and in regions with low-intensity variation it makes good smoothing, for example, in the interval [386, 398]. The other smoothing algorithms generate a loss of information at the edges and attenuate their intensity, causing some of them to be eliminated, for example in the intervals [260, 272] and [320, 335].

Based on the elements mentioned above, the initial alternative to smooth the images by preserving the edge information corresponds to the nonlinear anisotropic diffusion algorithm cEED.

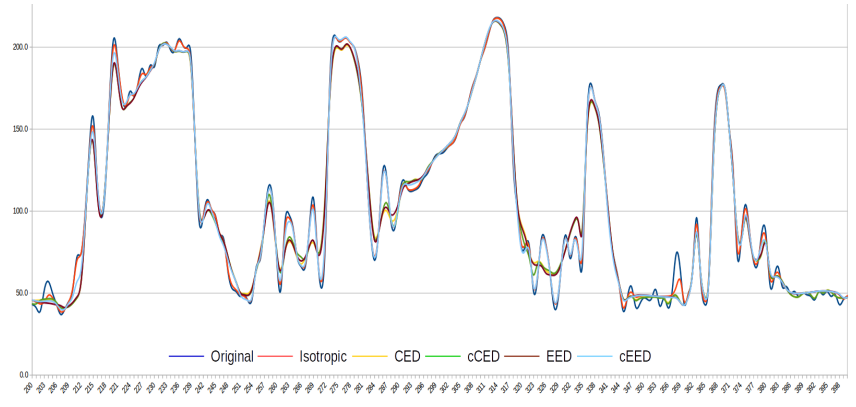

Figure 9: Profile behaviour of smoothing algorithms using lena image.

\section{$5.23 \mathrm{D}$ Case}

For the tests with 3D images were selected ten CT images of head-neck. It is proceeded in a similar way to the $2 \mathrm{D}$ case, ie, the original images are used first to evaluate the quality measures and to apply the smoothing algorithms in order to identify which stopping criterion performs better. Contrast-enhanced images are then used to identify if there is any change in the behaviour of the smoothing algorithms and in the stopping criteria. 


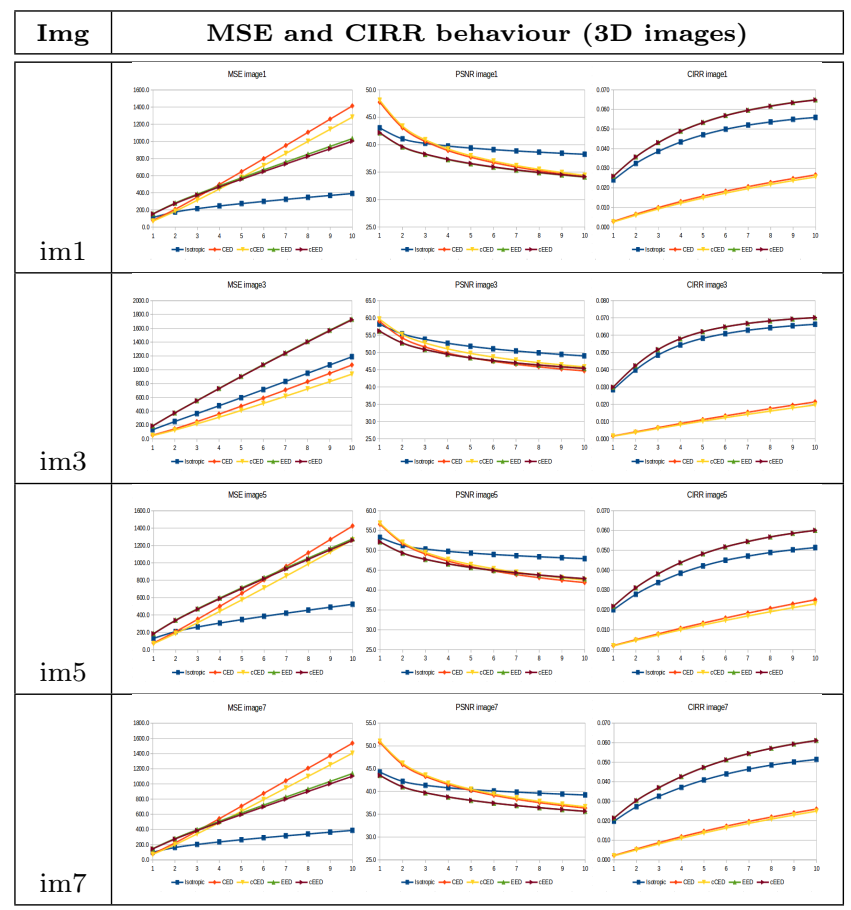

Table 7: MSE, PSNR and CIRR of 3D smoothed images.

Table 7 shows the behaviour of the MSE, PSNR and CIRR quality measures for images $1,3,5$ and 7 . The algorithms to be selected are those that generate the lowest value for the MSE measure and a higher value for PSNR and CIRR measures. However, it should be considered that as the image is smoothed the value of the PSNR measure decreases, for that reason, the EED and cEED algorithms present a lower value than the other algorithms.

The number of iterations defined by each of the stopping criteria for the ten test images is presented in Table 8. As can be seen, isotropic diffusion is similar using the two stopping criteria except for image 3 and image4. In the case of the EED and cEED diffusion, the number of iterations defined by $\mathrm{BF}$ criterion is half the number of iterations required by the JGTS criterion. JGTS stopping criterion generates a greater number of iterations required to stop the diffusion in all 3D images with respect to $\mathrm{BF}$ criterion. In addition, the number of iterations defined by the $\mathrm{BF}$ criterion is the same for the isotropic, EED and cEED diffusion algorithms.

Table 9 shows the images obtained from the smoothing process using stopping criteria JGTS and BF respectively. Visually the differences between the images are not perceptible. To see the impact of the number of iterations in the diffusion algorithms, the edges of the image 1 for the images generated by each of them were calculated. The algorithm proposed by Canny was used for this purpose. As the largest variation in the number of iterations was presented in the EED and cEED diffusion algorithms, it is expected that there is a significant variation in the edges.

\begin{tabular}{|c|c|c|c|c|c|c|}
\hline Img & Index & Isotr & CED & cCED & EED & cEED \\
\hline \hline \multirow{2}{*}{ im1 } & JGTS & 5 & 10 & 10 & 8 & 8 \\
\cline { 2 - 7 } & $\mathrm{BF}$ & 4 & 7 & 7 & 4 & 4 \\
\hline \multirow{2}{*}{ im2 } & $\mathrm{JGTS}$ & 5 & 10 & 10 & 8 & 8 \\
\cline { 2 - 7 } & $\mathrm{BF}$ & 4 & 7 & 7 & 4 & 4 \\
\hline \multirow{2}{*}{ im3 } & $\mathrm{JGTS}$ & 10 & 10 & 10 & 9 & 9 \\
\cline { 2 - 7 } & $\mathrm{BF}$ & 4 & 9 & 9 & 4 & 4 \\
\hline \multirow{2}{*}{ im4 } & $\mathrm{JGTS}$ & 10 & 10 & 10 & 10 & 10 \\
\cline { 2 - 7 } & $\mathrm{BF}$ & 4 & 9 & 9 & 4 & 4 \\
\hline \multirow{3}{*}{ im5 } & $\mathrm{JGTS}$ & 5 & 10 & 10 & 8 & 8 \\
\cline { 2 - 7 } & $\mathrm{BF}$ & 4 & 8 & 8 & 4 & 4 \\
\hline \multirow{2}{*}{ im6 } & $\mathrm{JGTS}$ & 5 & 10 & 10 & 8 & 8 \\
\cline { 2 - 7 } & $\mathrm{BF}$ & 4 & 8 & 8 & 4 & 4 \\
\hline \multirow{2}{*}{ im7 } & $\mathrm{JGTS}$ & 5 & 10 & 10 & 9 & 9 \\
\cline { 2 - 7 } & $\mathrm{BF}$ & 4 & 8 & 8 & 4 & 4 \\
\hline \multirow{2}{*}{ im8 } & $\mathrm{JGTS}$ & 5 & 10 & 10 & 9 & 9 \\
\cline { 2 - 7 } & $\mathrm{BF}$ & 4 & 8 & 8 & 4 & 4 \\
\hline \multirow{2}{*}{ im9 } & $\mathrm{JGTS}$ & 6 & 10 & 10 & 9 & 9 \\
\cline { 2 - 7 } & $\mathrm{BF}$ & 4 & 6 & 7 & 4 & 4 \\
\hline \multirow{2}{*}{ im10 } & $\mathrm{JGTS}$ & 6 & 10 & 10 & 9 & 9 \\
\cline { 2 - 7 } & $\mathrm{BF}$ & 4 & 6 & 7 & 4 & 4 \\
\hline
\end{tabular}

Table 8: Number of iterations using JGTS and BF stopping criteria.

The results of the obtained edges are presented in Table 10. In the rows is found each of the diffusion algorithms. In the second column the images obtained by using the JGTS stopping criterion for each diffusion algorithm. In the third column the images using the BF criterion. As can be seen, there is no variation in the edge detection in the images obtained using the EED and cEED diffusion by applying the two stopping criteria. In addition, isotropic diffusion presents results similar to EED and cEED diffusion. On the other hand, the diffusion CED and cCED allow detecting a greater number of edges with respect to the other three algorithms, independent of the stopping criterion used. This is due to the fact that the CED and cCED algorithms apply less smoothing in the internal regions of the image structures.

In conclusion, the edges detected in the images obtained using each one of the diffusion algorithms are equal independent of the stopping criterion used. Therefore, it is considered that the BF stopping criterion is more efficient than the JGTS criterion because it allows stopping the diffusion in a smaller number of iterations. 


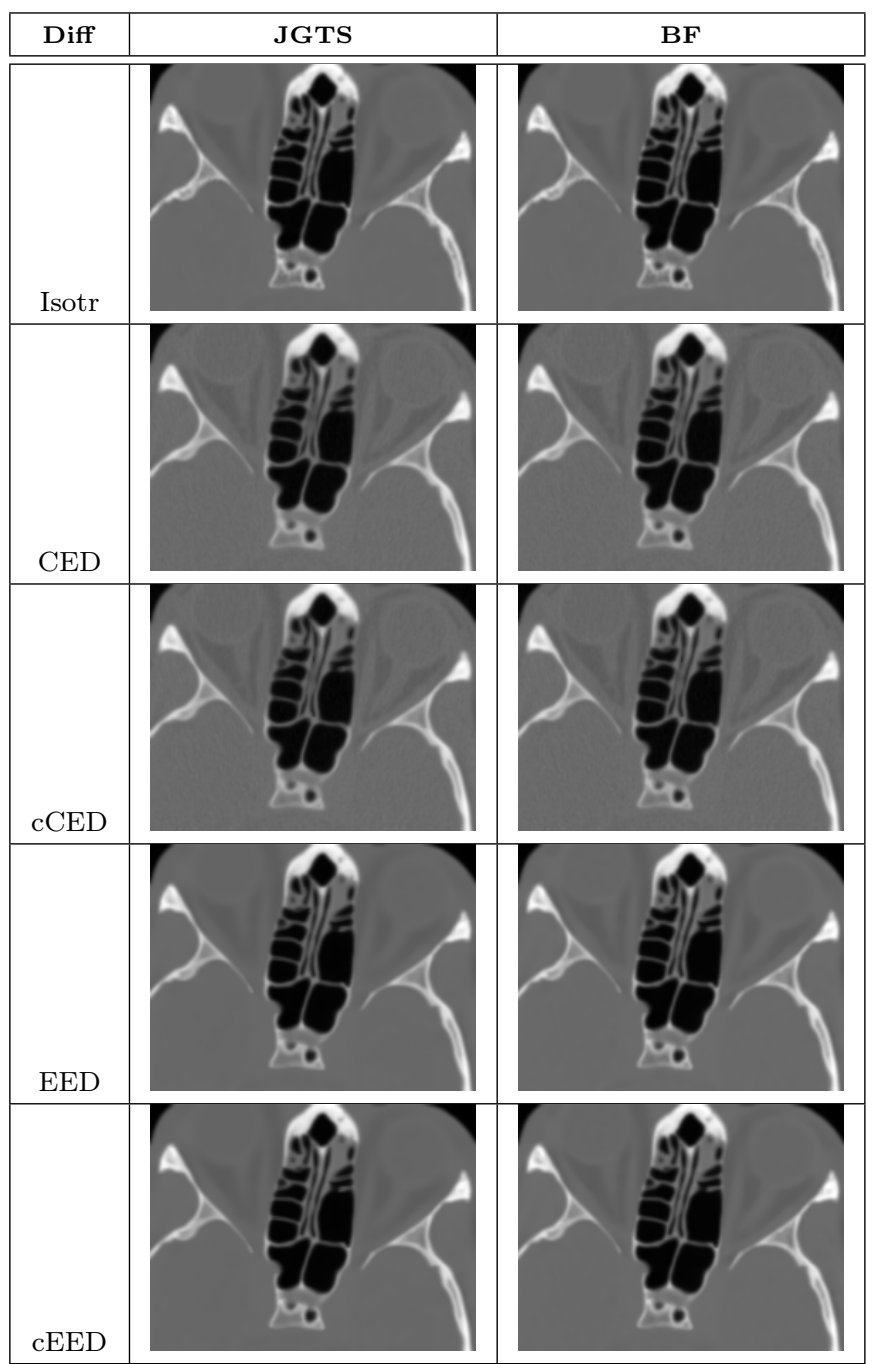

Table 9: 3D images smoothed using MSE and CIRR stopping criteria.

\section{CONCLUSIONS}

The selected nonlinear diffusion algorithms allowed to define that the edge information is preserved in a better way using the cEED algorithm. Isotropic algorithm also preserves the edges but in the internal regions of the structures does not perform a good smoothing. The CED and cCED algorithms do not properly preserve the edges and generate edges continuity incorrectly.

The proposed BF stopping criterion requires a lower number of iterations in 3D images. This is because the CIRR measure has an asymptotic behavior, while the MSE measure has a more linear behavior. This result allows to increase in automatic way the efficiency of the smoothing algorithms based on nonlinear anisotropic dif- fusion.

The stopping criterion $\mathrm{BF}$ is independent of the smoothing algorithm and it is not necessary to include it in the partial differential equation (PDE).

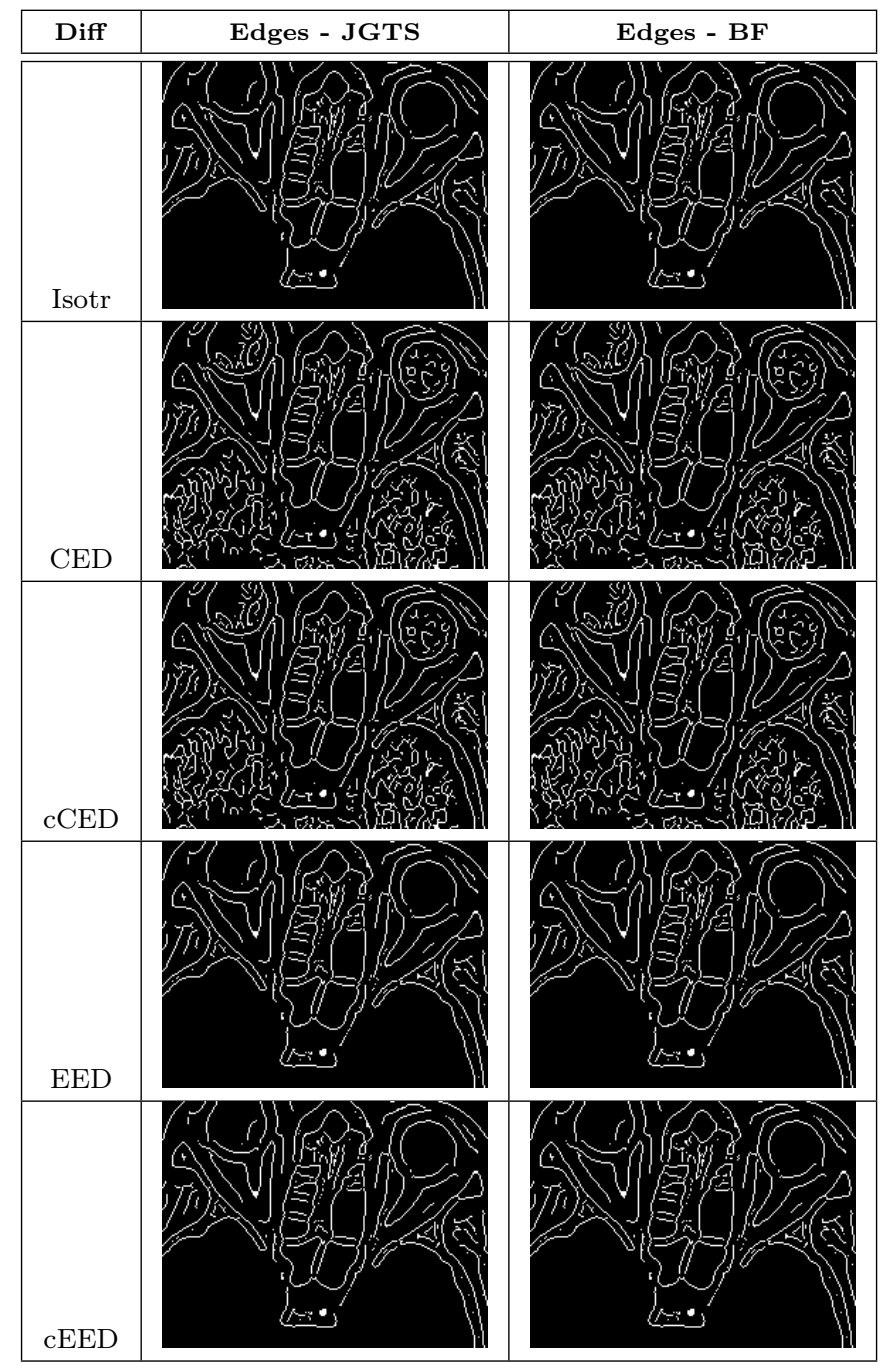

Table 10: Edges of 3D images smoothed.

\section{References}

[Afr2017] N. Afrin, W. Lai and N. Mohammed, "Performance Analysis of Corner Detection Algorithms Based on Edge Detectors," in WSCG2017 Proceedings, 2017, pp. $21-28$

[Aub2006] G. Aubert and P. Kornprobst, Mathematical Problems in Image Processing: Partial Differential Equations and the Calculus of Variations, Second. Springer Science+Business Media, LLC, 2006.

[Bar2014] T. Barbu, "Robust anisotropic diffusion scheme for image noise removal," Procedia Comput. Sci., vol. 35, no. C, pp. 522-530, 2014. 
[Baz2007] C. Bazán and P. Blomgren, "Image Smoothing and Edge Detection by Nonlinear Diffusion and Bilateral Filter," Res. Rep. CSRCR, vol. 21, 2007.

[Baz2009] C. Bazán, M. Miller, and P. Blomgren, "Structure enhancement diffusion and contour extraction for electron tomography of mitochondria," J. Struct. Biol., vol. 166 , no. 2 , pp. $144-155,2009$.

[Ber2000] M. Bertalmio, G. Sapiro, V. Caselles, and C. Ballester, "Image inpainting," Proc. Conf. Comput. Graph. Interact. Tech., pp. 417-424, 2000.

[Bro2006] T. Brox, J. Weickert, B. Burgeth, and P. Mrázek, "Nonlinear structure tensors," Image Vis. Comput., vol. 24, no. 1, pp. 41-55, 2006.

[Bus2019] C. Bustacara-Medina, L. Flórez-Valencia, An automatic stopping criterion for contrast enhancement using multi-scale top-hat transformation, To appear in Sensing and Imaging, 2019.

[Bus2016] C. Bustacara-Medina, M. Gomez-Mora and L. Flórez-Valencia, "Anisotropic Diffusion for Smoothing: A Comparative Study," Computer Vision and Graphics: International Conference, ICCVG 2016, Warsaw, Poland, September 19-21 Proceedings, pp. 109-120, 2016.

[Can1986] J. Canny, "A Computational Approach to Edge Detection," IEEE Trans. Pattern Anal. Mach. Intell., vol. PAMI-8, no. 6, pp. 679-698, 1986.

[Cao2003] F. Cao, Geometric Curve Evolution and Image Processing. Berlin Heidelberg: Springer-Verlag, 2003.

[Cap2001] I. Capuzzo Dolcetta and R. Ferretti, "Optimal stopping time formulation of adaptive image filtering," Appl. Math. Optim., vol. 43, no. 3, pp. 245-258, 2001.

[Cas1998] V. Caselles, J.-M. Morel, G. Sapiro, and A. Tannenbaum, "Introduction to the Special Issue on Partial Differential Equations and Geometry-Driven Diffusion in Image Processing and Analysis," IEEE Trans. Image Process., vol. 7, no. 3, pp. 269-273, 1998.

[Cha2010] S. M. Chao and D. M. Tsai, "Anisotropic diffusion with generalized diffusion coefficient function for defect detection in low-contrast surface images," Pattern Recognit., vol. 43, no. 5, pp. 1917-1931, 2010.

[Cot1993] G. H. Cottet and L. Germain, "Image-Processing Through Reaction Combined With Nonlinear Diffusion," Math. Comput., vol. 61, no. 204, pp. 659-673, 1993.

[Don2015] G. Dong, Z. Guo, Z. Zhou, D. Zhang, and B. Wo, "Coherence-enhancing diffusion with the source term," Appl. Math. Model., vol. 39, no. 19, pp. 60606072, 2015.

[Erd2012] E. Erdem, "Linear Diffusion," Ankara, 2012.

[Gil2004] G. Gilboa, N. Sochen, and Y. Y. Zeevi, "Image enhancement and denoising by complex diffusion processes," IEEE Trans. Pattern Anal. Mach. Intell., vol. 26, no. 8, pp. 1020-1036, 2004.
[Ily2010] A. Ilyevsky and E. Turkel, "Stopping criteria for anisotropic PDEs in image processing," J. Sci. Comput., vol. 45, no. 1-3, pp. 333-347, 2010.

[Joã2016] A. João, A. M. Gambaruto, J. Tiago, and A. Sequeira, "Computational advances applied to medical image processing: an update," Open Access Bioinformatics, vol. 8, pp. 1-15, 2016.

[Kee2002] S. L. Keeling and R. Stollberger, "Nonlinear anisotropic diffusion filtering for multiscale edge enhancement," Inverse Probl., vol. 18, no. 1, pp. 175190, 2002.

[Li2005] C. Li, C. Xu, C. Gui, and M. Fox, "Level Set Evolution without Re-Initialization: A New Variational Formulation," in 2005 IEEE Computer Society Conference on Computer Vision and Pattern Recognition (CVPR'05), 2005, vol. 1, pp. 430-436.

[Li2005a] X. Li, "The anatomy of anisotropic diffusion filters," Can. Soc. Explor. Geophys., vol. 30, no. 5, pp. 54-60, 2005.

[Men2009] A. M. Mendrik, E. J. Vonken, A. Rutten, M. A. Viergever, and B. Van Ginneken, "Noise reduction in computed tomography scans using 3-D anisotropic hybrid diffusion with continuous switch," IEEE Trans. Med. Imaging, vol. 28, no. 10, pp. 15851594, 2009.

[Mir2014] J.-M. Mirebeau, J. Fehrenbach, L. Risser, and S. Tobji, "Anisotropic Diffusion in ITK," Insight J., pp. $1-9,2014$.

[Mra2003] P. Mrázek and M. Navara, "Selection of optimal stopping time for nonlinear diffusion," Int. J. Comput. Vis., vol. 52, no. 2-3, pp. 189-203, 2003.

[Nit1992] M. Nitzberg and T. Shiota, "Nonlinear Image Filtering with Edge and Corner Enhancement," IEEE Transactions on Pattern Analysis and Machine Intelligence, vol. 14, no. 8. pp. 826-833, 1992.

[Per1990] P. Perona and J. Malik, "Scale-space and edge detection using anisotropic diffusion," IEEE Trans. Pattern Anal. Mach. Intell, vol. 12, no. 7, pp. 629-639, 1990.

[Per1990a] P. Perona and J. Malik, "Detecting and localizing edges composed of steps, peaks and roofs," in Proceedings Third International Conference on Computer Vision, 1990, pp. 52-57.

[Pra2016] S. Prasath, "Adaptive coherence-enhancing diffusion flow for color images," Inform., vol. 40, no. 3, pp. 337-342, 2016.

[Rud1989] L. Rudin and S. Osher, "Feature-Oriented Image Enhancement with Shock filters, I," 1989.

[Sap2006] G. Sapiro, Geometric Partial Differential Equations and Image Analysis. Cambridge University Press, 2006.

[Spo1999] J. Sporring and J. Weickert, "Information Measures in Scale Spaces," IEEE Trans. Inf. Theory, vol. 45, no. 3, pp. 1051-1058, 1999. 
[Ter1994] B. M. ter Haar Romeny, Geometry-Driven Diffusion in Computer Vision, vol. 1. Springer-Science and Business Media, 1994.

[Tsi2013] C. Tsiotsios and M. Petrou, "On the choice of the parameters for anisotropic diffusion in image processing," Pattern Recognit., vol. 46, no. 5, pp. 1369-1381, 2013.

[Wei1996] J. Weickert, "Theoretical Foundations of Anisotropic Diffusion in Image Processing," Comput. Suppl., vol. 11, pp. 221-236, 1996.
[Wei1997] J. Weickert, S. Ishikawa, and A. Imiya, "Scale-Space has been Discovered in Japan," 1997.

[Wei1998] J. Weickert, Anisotropic diffusion in image processing, vol. 256, no. 3. B.G. Teubner Stuttgart, 1998.

[Wei1999] J. Weickert, "Coherence-Enhancing Diffusion Filtering," Int. J. Comput. Vis., vol. 31, no. 2, pp. 1-23, 1999.

[Wei2002] J. Weickert and H. Scharr, "A Scheme for CoherenceEnhancing Diffusion Filtering with Optimized Rotation Invariance," J. Vis. Commun. Image Represent., vol. 13, no. 1-2, pp. 103-118, 2002. 